\title{
Advanced Imaging for Precision Medicine in Breast Cancer: From Morphology to Function
}

\author{
Katja Pinker ${ }^{a, b}$ \\ a Department of Radiology, Breast Imaging Service, Memorial Sloan Kettering Cancer Center, New York, NY, USA; \\ ${ }^{b}$ Department of Biomedical Imaging and Image-Guided Therapy, Division of Molecular and Gender Imaging, \\ Medical University of Vienna, Vienna, Austria
}

Breast cancer is the most common cancer in women, the 2nd leading cause of female cancer deaths and thus remains a major medical and socio-economic burden. Medical imaging has always been an integral part in breast cancer care ranging from diagnosis and staging to therapy monitoring and post-therapeutic follow-up [1-5]. Screening mammography has significantly contributed to the detection of breast cancer especially at an early, prognostically favorable stage and mammography has been demonstrated to reduce breast cancer mortality [6-9]. Mammography is relatively inexpensive and widely available. However, its sensitivity is limited, ranging from 70 to $85 \%$ [10-12] overall but is significantly reduced to $30-50 \%$ in women with dense breasts, which can be attributed to the 'masking effect' of dense breast tissue. This effect is due to overlap with normal breast tissue thus obscuring breast lesions and is most pronounced in extremely dense breast parenchyma [13, 14]. Supplemental imaging examinations to overcome the limitation in sensitivity traditionally includes screening ultrasound, and combined mammography and ultrasound can improve sensitivities for breast cancer detection to $91 \%[10,15]$.

Recently, digital breast tomosynthesis (DBT) has been introduced. DBT is an imaging technique that in contrast to mammography allows the 3-dimensional (3D) evaluation through the volumetric reconstruction of the whole breast from a finite number of low-dose $2 \mathrm{D}$ projections obtained by different X-ray tube angles. Thereby DBT minimizes the effect of overlapping breast tissue and significantly improves lesion detection. Several studies have consistently shown that DBT detects up to $50 \%$ more cancers and it can be expected that DBT will eventually replace mammography in in breast imaging and screening [16-27].

Although DBT and ultrasound can increase cancer detection, there are still limitations in breast cancer detection, prognosis, and prediction. Medical research has entered the genomic era, aiming to devise precise cancer therapies targeting the underlying oncogenic mechanisms. Genetic profiling has revolutionized breast cancer classifications, with molecular tumor subtypes being unevenly distributed among women with breast cancer [28-30]. Molecular subtypes are not only associated with different tumor phenotypes but also with distinct variations in response to therapy and patient survival. Accordingly, subtype-based recommendations for systemic therapies have now been implemented in clinical practice [28-31]. To match advanced therapeutic strategies in the era of precision medicine, diagnostic tests must be equally multilayered and complex to identify the underlying functional processes of cancer development and progression.

However, with conventional imaging methods such as mammography, DBT, and sonography, which are qualitative tests that detect morphologic, non-specific tissue changes, the assessment and comprehension of breast cancer in all its diversity is not possible. To overcome these limitations, functional imaging techniques to improve breast cancer diagnosis have been explored. In this context, magnetic resonance imaging (MRI) has emerged as an exceptionally powerful, versatile, and precise imaging technique. MRI is an essential non-invasive technique in breast imaging with multiple established indications, such as pre-operative staging of breast cancer, evaluation of therapy response in patients receiving neoadjuvant treatment, differentiation between scar tissue and tumor recurrence, examination of patients with breast implants, and screening of high-risk patients $[1,5]$. Dynamic contrast-enhanced MRI of the breast provides both high-resolution morphologic and functional quantitative information on neovascularity as a tumor-specific feature [32-35]. MRI is the most sensitive exam for the detection of breast cancer in women at any given risk [32-36] and often detects cancers that are occult in mammography, DBT and sonography $[37,38]$. Due to these excellent results specific screening programs for high-risk women that include both annual mammography and MRI have been developed [32,39] and there is discussion about screening of women at average of risk cancer with abbreviated MRI protocols [40-42]. In addition to breast cancer detection there is evidence that quantitative dynamic contrast enhanced (DCE)-MRI models are useful in the assessment and prediction of

\section{KARGER}

(C) 2017 S. Karger GmbH, Freiburg

Fax +497614520714
Katja Pinker, MD, PhD

Breast Imaging Service, Department of Radiology

Memorial Sloan Kettering Cancer Center

300 E 66th Street, New York, NY 10065, USA

pinkerdk@mskcc.org 
response to neo-adjuvant chemotherapy (NAC) [43, 44]. However, despite these excellent results for breast cancer detection and prediction of treatment response, MRI may not be an option for every woman, due to either lacking availability or contra-indications to MRI, such as metallic implants, allergic reactions to MRI contrast agents, or severe claustrophobia.

Contrast enhanced digital mammography (CEDM) is a novel mammography technology which, similar to MRI, is based on the principle of imaging neovascularity. CEDM uses intravenous iodinated contrast medium to detect breast cancer on a digital mammography platform. Compared to MRI, CEDM is relatively inexpensive and potentially more accessible to women. Several studies in the diagnostic setting have demonstrated that CEDM is significantly more sensitive and specific than mammography alone in women with abnormal screening mammograms, ultrasound, clinical symptoms, and known cancer. Early studies have even demonstrated that the performance of CEDM is superior to that of mammography alone in the diagnostic setting, especially in women with dense breast tissue [45] and comparable to the performance of MRI in women with known cancers [46-50].

The current issue of BREAST CARE with focus on 'Imaging in Breast Cancer' will provide a comprehensive summary of the re- cent advances and current possibilities in clinical breast imaging. The added value of DBT to mammography in BI-RADS assessment and follow-up management is investigated by Galati et al. [51]. Knogler et al. [52] present results on CEDM for breast lesion detection, characterization and comparison to DCE-MRI. The potential of DCE-MRI including quantitative pharmacokinetic modeling for the assessment and prediction of response to neoadjuvant chemotherapy in breast cancer overall (Dietzel et al. [53]) and with a special focus on inflammatory breast cancer (van Uden et al. [54]) is discussed. In addition, Clauser et al. [55] provide data on the usefulness of short-term imaging follow-up in patients with BIRADS 3 and $4 \mathrm{a}$ lesions and the benign histopathologic diagnosis of a fibroadenoma at image-guided biopsy.

The field of breast imaging has moved beyond morphology towards function and is a still evolving. More significant advances are expected, which will further aid the development of novel approaches for precision medicine in breast cancer.

\section{Disclosure Statement}

The author has nothing to disclose.

\section{References}

1 Mann RM, Balleyguier C, Baltzer PA, et al.: Breast MRI: EUSOBI recommendations for women's information. Eur Radiol 2015;25:3669-3678.

2 Sardanelli F, Helbich TH, European Society of Breast Imaging: Mammography: EUSOBI recommendations for women's information. Insights into imaging. 2012; 3.7-10.

3 Sardanelli F, Aase HS, Alvarez M, et al.: Position paper on screening for breast cancer by the European Society of Breast Imaging (EUSOBI) and 30 national breast radiology bodies from Austria, Belgium, Bosnia and Herzegovina, Bulgaria, Croatia, Czech Republic, Denmark, Estonia, Finland, France, Germany, Greece, Hungary, Iceland, Ireland, Italy, Israel, Lithuania, Moldova, The Netherlands, Norway, Poland, Portugal, Romania, Serbia, Slovakia, Spain, Sweden, Switzerland and Turkey. Eur Radiol 2017;27:2737-2743.

4 D’Orsi CJ, Sickles EA, Mendelson EB, Morris EA, et al.: ACR BI-RADS ${ }^{\circledR}$ Atlas, Breast Imaging Reporting and Data System (ed 5). Reston, VA, American College of Radiology, 2013.

5 Sardanelli F, Boetes C, Borisch B, et al.: Magnetic resonance imaging of the breast: recommendations from the EUSOMA working group. European journal of cancer. 2010;46:1296-1316.

6 Gabe R, Duffy SW: Evaluation of service screening mammography in practice: the impact on breast cancer mortality. Ann Oncol 2005;16 (Suppl 2):ii153-ii162.

7 Broeders M, Moss S, Nystrom L, et al.: The impact of mammographic screening on breast cancer mortality in Europe: a review of observational studies. J Med Screen 2012;19 (suppl 1):14-25.

8 Moss SM, Nystrom L, Jonsson H, et al.: The impact of mammographic screening on breast cancer mortality in Europe: a review of trend studies. J Med Screen 2012;19 (suppl 1):26-32.
9 Massat NJ, Dibden A, Parmar D, et al.: Impact of screening on breast cancer mortality: The UK program 20 years on. Cancer Epidemiol Biomarkers Prev 2016; 25:455-462.

10 Zonderland HM, Coerkamp EG, Hermans J, et al.: Diagnosis of breast cancer: contribution of US as an adjunct to mammography. Radiology 1999;213:413-422.

11 Kerlikowske K, Carney PA, Geller B, et al.: Performance of screening mammography among women with and without a first-degree relative with breast cancer. Ann Intern Med 2000;133:855-863.

12 Warner E, Plewes DB, Hill KA, et al.: Surveillance of BRCA1 and BRCA2 mutation carriers with magnetic resonance imaging, ultrasound, mammography, and clinical breast examination. JAMA 2004;292:1317-1325.

13 Jackson VP, Hendrick RE, Feig SA, et al.: Imaging of the radiographically dense breast. Radiology 1993;188: 297-301.

14 Rhodes DJ, Radecki Breitkopf C, Ziegenfuss JY, et al.: Awareness of breast density and its impact on breast cancer detection and risk. J Clin Oncol 2015;33:11431150.

15 Schaefer FK, Waldmann A, Katalinic A, et al.: Influence of additional breast ultrasound on cancer detection in a cohort study for quality assurance in breast diagnosis-analysis of 102,577 diagnostic procedures. Europ Radiol 2010;20:1085-1092.

16 Freer PE, Riegert J, Eisenmenger L, et al.: Clinical implementation of synthesized mammography with digital breast tomosynthesis in a routine clinical practice. Breast Cancer Res Treat 2017; doi: 10.1007/s10549017-4431-1.

17 Yun SJ, Ryu CW, Rhee SJ, et al.: Benefit of adding digital breast tomosynthesis to digital mammography for breast cancer screening focused on cancer characteristics: a meta-analysis. Breast Cancer Res Treat 2017; 164:557-569
18 Friedewald SM: Breast tomosynthesis: practical considerations. Radiol Clin North Am 2017;55:493-502.

19 Poplack S: Breast Tomosynthesis: clinical evidence. Radiol Clin North Am 2017;55:475-492.

20 Skaane P: Breast cancer screening with digital breast tomosynthesis. Breast Cancer 2017;24:32-41.

21 Conant EF, Beaber EF, Sprague BL, et al.: Breast cancer screening using tomosynthesis in combination with digital mammography compared to digital mammography alone: a cohort study within the PROSPR consortium. Breast Cancer Res Treat 2016;156:109-116.

22 Friedewald SM, Rafferty EA, Rose SL, et al.: Breast cancer screening using tomosynthesis in combination with digital mammography. JAMA 2014;311:2499-2507.

23 Pisano ED, Yaffe MJ: Breast cancer screening: should tomosynthesis replace digital mammography? JAMA 2014;311:2488-2489.

24 Haas BM, Kalra V, Geisel J, Raghu M, Durand M, Philpotts LE: Comparison of tomosynthesis plus digital mammography and digital mammography alone for breast cancer screening. Radiology 2013;269:694-700.

25 Ciatto S, Houssami N, Bernardi D, et al.: Integration of 3D digital mammography with tomosynthesis for population breast-cancer screening (STORM): a prospective comparison study. Lancet Oncol 2013;14:583-589.

26 Melnikow J, Fenton JJ, Whitlock EP, et al.: U.S. Preventive Services Task Force Evidence Syntheses, formerly Systematic Evidence Reviews. Supplemental Screening for Breast Cancer in Women with Dense Breasts: A Systematic Review for the US Preventive Service Task Force. Rockville (MD), Agency for Healthcare Research and Quality (US), 2016.

27 Clauser P, Nagl G, Helbich TH, et al.: Diagnostic performance of digital breast tomosynthesis with a wide scan angle compared to full-field digital mammography for the detection and characterization of microcalcifications. Eur J Radiol 2016;85:2161-2168. 
28 Mazurowski MA, Zhang J, Grimm LJ, Yoon SC, Silber JI: Radiogenomic analysis of breast cancer: luminal B molecular subtype is associated with enhancement dynamics at MR imaging. Radiology 2014;273:365-372.

29 Huber KE, Carey LA, Wazer DE: Breast cancer molecular subtypes in patients with locally advanced disease: impact on prognosis, patterns of recurrence, and response to therapy. Semin Radiat Oncol 2009;19:204210.

30 Lam SW, Jimenez CR, Boven E: Breast cancer classification by proteomic technologies: current state of knowledge. Cancer Treat Rev 2014;40:129-138.

31 Carey LA, Perou CM, Livasy CA, et al.: Race, breas cancer subtypes, and survival in the Carolina Breast Cancer Study. Jama 2006;295:2492-2502.

32 Mann RM, Balleyguier C, Baltzer PA, et al.: Breast MRI: EUSOBI recommendations for women's information. Eur Radiol 2015;25:3669-3678.

33 Kuhl C, Weigel S, Schrading S, et al.: Prospective multicenter cohort study to refine management recommen dations for women at elevated familial risk of breast cancer: the EVA trial. J Clin Oncol 2010;28:1450-1457.

34 Rijnsburger AJ, Obdeijn IM, Kaas R, et al.: BRCA1associated breast cancers present differently from BRCA2-associated and familial cases: long-term follow-up of the Dutch MRISC Screening Study. J Clin Oncol 2010;28:5265-5273

35 Riedl CC, Luft N, Bernhart C, et al.: Triple-modality screening trial for familial breast cancer underlines the importance of magnetic resonance imaging and questions the role of mammography and ultrasound regardless of patient mutation status, age, and breast density. J Clin Oncol 2015;33:1128-1135.

36 Obdeijn IM, Winter-Warnars GA, Mann RM, et al.: Should we screen BRCA1 mutation carriers only with MRI? A multicenter study. Breast Cancer Res Treat 2014;144:577-582.

37 Houssami N, Ciatto S, Macaskill P, et al.: Accuracy and surgical impact of magnetic resonance imaging in breast cancer staging: systematic review and metaanalysis in detection of multifocal and multicentric cancer. J Clin Oncol 2008;26:3248-3258.
38 Lo G, Scaranelo AM, Aboras H, et al.: Evaluation of the utility of screening mammography for high-risk women undergoing screening breast MR imaging. Radiology 2017: doi: 10.1148/radiol.2017161103.

39 Saslow D, Boetes C, Burke W, et al.: American Cancer Society guidelines for breast screening with MRI as an adjunct to mammography. CA Cancer J Clin 2007;57: 75-89.

40 Kuhl CK.: Abbreviated breast MRI for screening women with dense breast: the EA1141 trial. Br J Radiol 2017: doi: 10.1259/bjr.20170441.

41 Kuhl CK, Schrading S, Strobel K, et al.: Abbreviated breast magnetic resonance imaging (MRI): first postcontrast subtracted images and maximum-intensity projection-a novel approach to breast cancer screening with MRI. J Clin Oncol 2014;32:2304-2310.

42 Mango VL, Morris EA, David Dershaw D, et al. Abbreviated protocol for breast MRI: are multiple sequences needed for cancer detection? Eur J Radiol 2015;84:65-70.

43 Minarikova L, Bogner W, Pinker K, et al.: Investigating the prediction value of multiparametric magnetic resonance imaging at $3 \mathrm{~T}$ in response to neoadjuvant chemotherapy in breast cancer. Eur Radiol 2017;27:1901-1911.

44 Marinovich ML, Houssami N, Macaskill P, et al.: Meta-analysis of magnetic resonance imaging in detecting residual breast cancer after neoadjuvant therapy. J Natl Cancer Inst 2013;105:321-333.

45 Cheung YC, Lin YC, Wan YL, et al.: Diagnostic performance of dual-energy contrast-enhanced subtracted mammography in dense breasts compared to mammography alone: interobserver blind-reading analysis. Eur Radiol 2014;24:2394-2403.

46 Lobbes MB, Lalji U, Houwers J, et al.: Contrast-enhanced spectral mammography in patients referred from the breast cancer screening program. Eur Radiol 2014;24:1668-1676.
47 Dromain C, Balleyguier C, Adler G, et al.: Contrastenhanced digital mammography. Eur J Radiol 2009;69: 34-42.

48 Jochelson MS, Dershaw DD, Sung JS, et al. Bilateral contrast-enhanced dual-energy digital mammography: feasibility and comparison with conventional digital mammography and MR imaging in women with known breast carcinoma. Radiology 2013;266:743-751.

49 Dromain C, Thibault F, Muller S, et al.: Dual-energy contrast-enhanced digital mammography: initial clinical results. European radiology. 2011;21[3]:565-74.

50 Fallenberg EM, Dromain C, Diekmann F, et al. Contrast-enhanced spectral mammography versus MRI: Initial results in the detection of breast cancer and assessment of tumour size. Eur Radiol 2014;24:256-264.

51 Galati F, Marzocca F, Bassetti E, et. al.: Added value of digital breast tomosynthesis combined with digital mammography according to reader agreement: changes in BI-RADS rate and follow-up management Breast Care 2017;DOI: 10.1159/000477537.

52 Knogler T, Homolka P, Hoernig M, et al.: Application of BI-RADS descriptors in contrast-enhanced dualenergy mammography: comparison with MRI. Breast Care 2017; DOI: 10.1159/000478899.

53 Dietzel M, Kaiser C, Pinker K, et. al.: Automated semiquantitative analysis of breast MRI: potential imaging biomarker for the prediction of tissue response to neoadjuvant chemotherapy. Breast Care 2017;DOI: $10.1159 / 000480226$.

54 van Uden DJP, de Wilt JHW, Meeuwis C, et. al.: Dynamic contrast-enhanced magnetic resonance imaging in the assessment of inflammatory breast cancer prior to and after neoadjuvant treatment. Breast Care 2017;DOI: 10.1159/000475745.

55 Clauser P, Bazzocchi M, Marcon M, et. al.: Results of short-term follow-up in BI-RADS 3 and 4a breast lesions with a histological diagnosis of fibroadenoma at percutaneous needle biopsy. Breast Care 2017;DOI: $10.1159 / 000477536$. 